\title{
Metaboliten-FISHing
}

\section{Die chemische Sprache von Symbiosen sichtbar machen}

BENEDIKT GEIER, MANUEL LIEBEKE

MAX-PLANCK-INSTITUT FÜR MARINE MIKROBIOLOGIE, BREMEN

\section{Small molecules - metabolites - provide the basis for chemical inter- actions between hosts and microbes. Especially in animal-microbe symbioses, the close physical interactions require a spatial organiza- tion of cells and their metabolites. Correlative mass spectrometry imaging (MSI) and fluorescence microscopy provide powerful tools to address the technical challenge of linking metabolite production to symbiont and host cells in mixed communities and study spatial metabolomes of symbioses in situ.}

DOI: $10.1007 / \mathrm{s} 12268-020-1435-\mathrm{x}$

(C) Die Autoren 2020

Um in der Natur zu bestehen, leben Bakterien und Tiere oft in engem Zusammenspiel und leben in Symbiosen. Die Grundlage jeder Interaktion zwischen Bakterien und Tieren ist eine gemeinsame chemische Sprache. Diese Sprache basiert primär auf kleinen Molekülen (unter 1.500 Da), den Metaboliten, mittels derer sich die Partner erkennen, verteidigen, miteinander kommunizieren und gegenseitig manipulieren (Abb. 1, [1]).

Metaboliten werden meist mittels Massenspektrometrie (MS) analysiert, indem das Gewebe samt assoziierter Bakterien homogenisiert wird, die Metaboliten extrahiert und anschließend gemessen werden. Dabei können hunderte bis tausende Metaboliten, das Metabolom, aus nur einem Extrakt erfasst werden.

Zellextrakte führen jedoch zu einem grundlegenden Problem: Die Anordnung der Wirtszellen, Bakterien und Metaboliten - das räumliche Metabolom - wird zerstört. Somit können einzelne Metaboliten nicht mehr ihrem Produktions- und Wirkungsort innerhalb der Symbiose zugeordnet werden. Um zu verstehen, wie Symbiosen aufgebaut sind und funktionieren, benötigen wir eine Methode, die uns erlaubt, das räumliche Metabolom von Wirts- und Bakterienzellen zu messen. Während standardisierte histologische Färbungen und Fluoreszenzmarker die Mikroskopie von Tier- und Bakterienzellen ermögli- chen, stellt die Visualisierung einzelner Metaboliten eine größere Herausforderung dar.

Gute Möglichkeiten, um Metabolitenverteilungen zu visualisieren, bietet die bildgebende Massenspektrometrie (mass spectrometry imaging, MSI), besonders in Verbindung mit Matrix-unterstützter Laser-Desorption/Ionisation (matrix-assisted laser desorption/ionization, MALDI), also eine Art Hybrid

\section{Ein molekulares Mikroskop}

aus Mikroskopie und MS - ein molecular microscope [2]. Dabei werden die Metaboliten von der Probenoberfläche mit einem Laser Punkt für Punkt ionisiert, mit MS gemessen und das Punktraster anschließend als Ionenbild dargestellt [3]. Durch Annotationsplattformen wie METASPACE [4] lassen sich die MSI-Daten mit Metabolitendatenbanken abgleichen und die Ionen(bilder) bestimmten Metaboliten zuordnen.

Die Kombination von MALDI-MSI und Mikroskopie ist also eine vielversprechende Methode, Bakterien in Wirtsgeweben und deren Metaboliten zu untersuchen. Eine viel größere Herausforderung ist die korrelative Anwendung beider Techniken auf derselben Probenoberfläche. Um Metaboliten abzubilden, bietet das Schockfrieren von frischem Gewebe die beste chemische Fixierung. Zellspezifische Marker sollten erst nach MALDIMSI angewandt werden, da diese das Metabolom verändern. Wird MALDI-MSI zuerst durchgeführt, kann der Laser durch „Einschusslöcher" das Gewebe beschädigen und anschließende Markierungsansätze erschweren

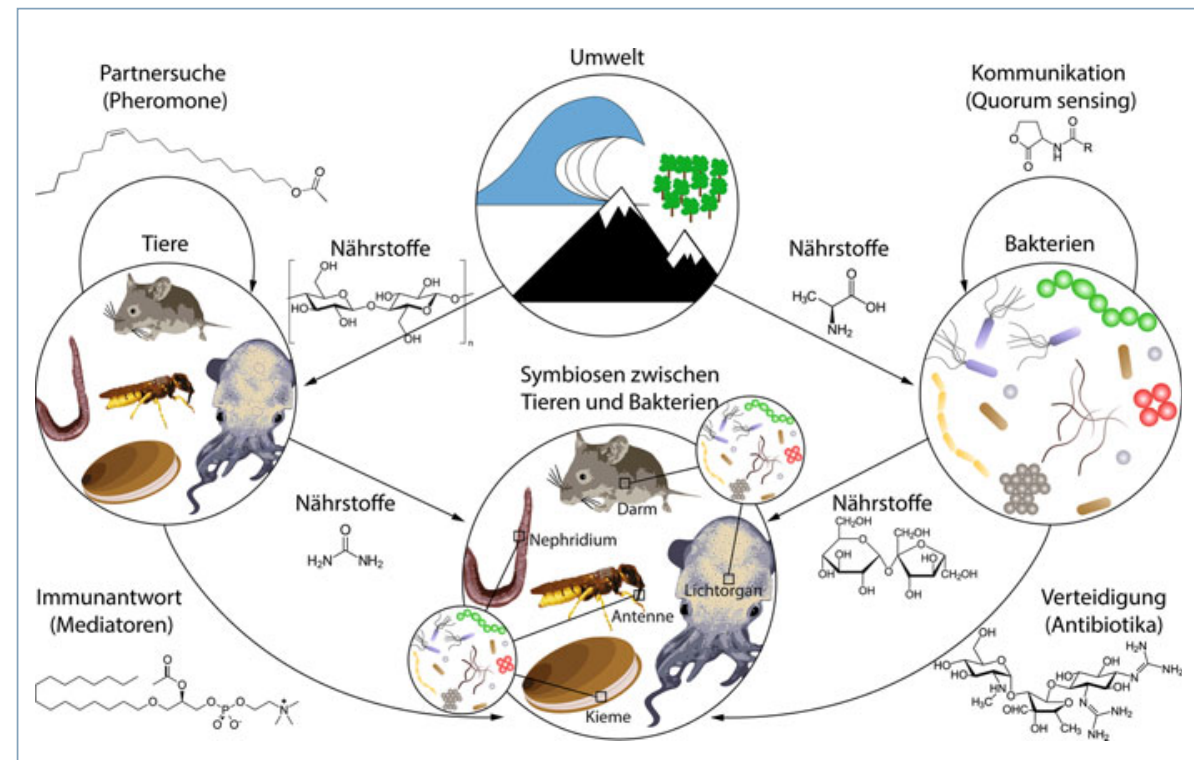

$\Delta$ Abb. 1: Beispiele für Metaboliten, die chemische Interaktionen zwischen Bakterien, Tieren und der Umwelt ermöglichen und den Bakterien erlauben, die verschiedenen Wirtsorgane zu besiedeln. 


\section{MALDI-MSI und Mikroskopie in Einklang bringen}

Einen Fortschritt bei der Untersuchung kultivierbarer Symbiosen lieferten William J. Perry et al. (2019): Sie kombinierten MALDIMSI mit Fluoreszenzmikroskopie, indem sie Bakterien mit grün fluoreszierendem Protein (GFP) transfizierten und damit ein Mausmodell infizierten, um die Fluoreszenzmarker vor dem Schockfrieren einzubringen [5]. Leider können die meisten Symbiosen nicht im Labor kultiviert werden. Es bedarf daher einer anderen Strategie, um Bakterien und ihre Metaboliten sichtbar zu machen.

Fluoreszenz-in-situ-Hybridisierung (FISH) ist die gängige Methode, nicht-kultivierbare Bakterien mittels Fluoreszenzmarkern taxonomisch zu identifizieren [6]. Martin Kaltenpoth et al. (2016) zeigten durch korrelatives MSI und FISH, wie symbiotische Bakterien die Kokons ihrer Insektenwirte durch Antibiotikaproduktion vor Pilzbefall schützen [7]. Dabei wurde deutlich, dass die destruktiven Eigenschaften des MALDI-Lasers den korrelativen MALDI-FISH-Ansatz auf Pixelgrößen um 50 Mikrometer limitierten. Das ist zu gering, um einzelne tierische Zellen (etwa $50 \mu \mathrm{m}$ ) oder Bakterien (etwa $1 \mu \mathrm{m}$ ) abzubilden. Generell ist das Auflösungsvermögen kommerzieller MALDI-MSI-Geräte mit Pixelgrößen von 25 bis 5 Mikrometern bis zu zwei Größenordnungen geringer als das von Fluoreszenzmikroskopen mit etwa 0,25 Mikrometern pro Pixel. Um Metaboliten bestimmten Zellen und damit ihrem möglichen Produktionsort zuzuordnen, werden die Metabolitenund Fluoreszenzbilder abgeglichen [8]. Jeder MALDI-MSI-Pixel repräsentiert ein Massenspektrum. Das Problem: Bei einem zu großen Pixel wird die Metabolitenmessung dieses Spektrums über mehre Zellen interpoliert, selbst wenn sich das Metabolom der einzelnen Zellen unterscheidet. Folglich bestimmt die Methode mit der geringeren Auflösung, hier MALDI-MSI, wie genau man korrelative Daten analysieren und interpretieren kann.

\section{Schnappschuss von Molekülen in einer Tiefseesymbiose}

In unserer Studie [9] verbesserten wir mittels eines hochauflösenden MALDI-MSI-Geräts von Mario Kompauer et al. [10] und der Entwicklung eines passenden Protokolls korrelatives MALDI-FISH auf eine Auflösung von drei Mikrometern pro Pixel (Abb. 2). Dadurch konnten wir die Verteilung der Metaboliten einzelner Tierzellen mit MALDIMSI sichtbar machen und die Symbionten in
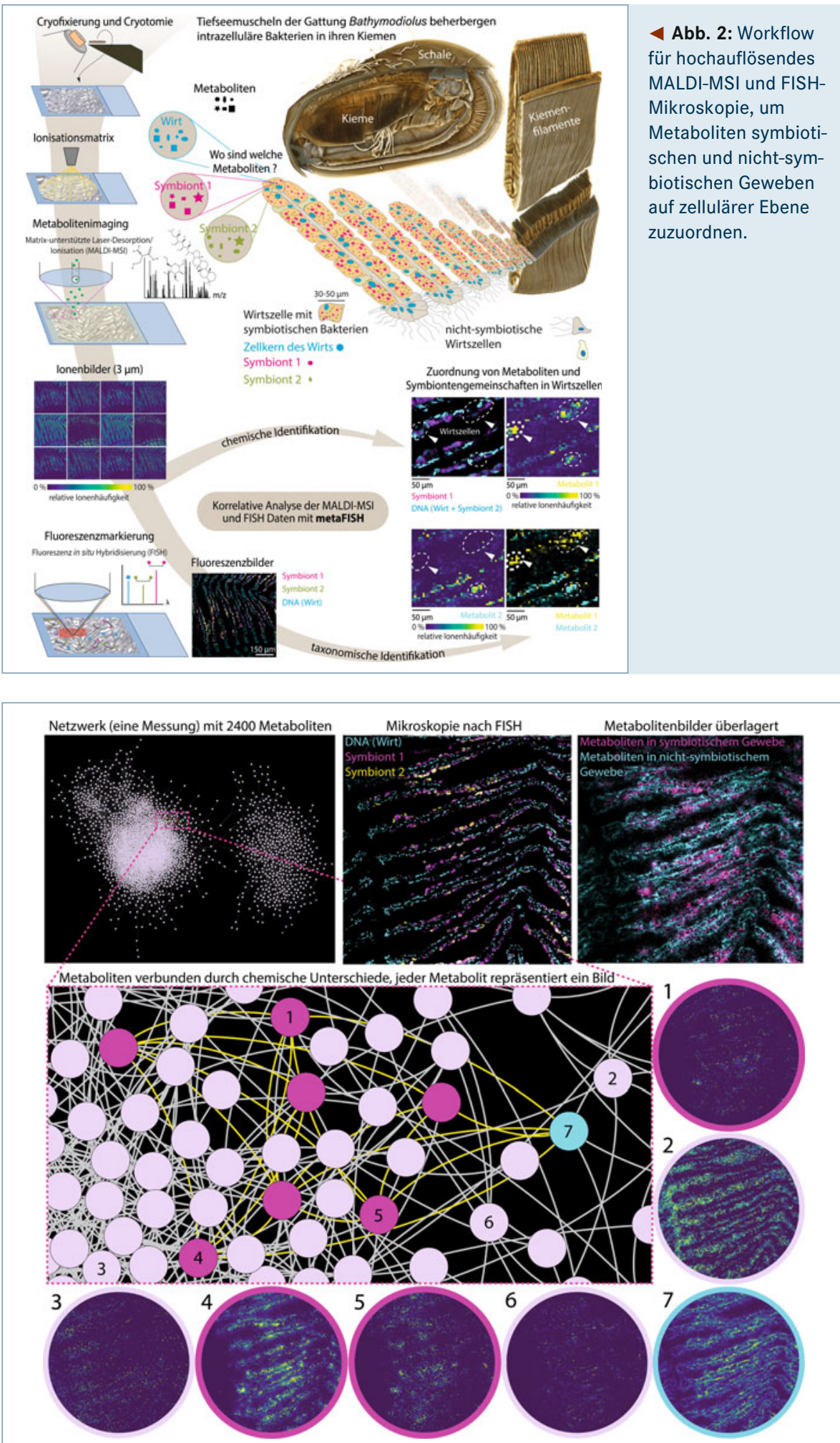

Abb. 3: Chemische Netzwerke ergänzen die Analyse der metaFISH-Bilddaten, indem sie potenziell chemisch verwandte Metaboliten vernetzen. Jeder Punkt repräsentiert einen Metabolit, hinter dem auch ein MALDI-MSI-Bild steckt. Die Verbindungen zwischen den Punkten (Metaboliten) sind theoretische Unterschiede der genauen Masse der Metaboliten. Unterscheiden sich beispielsweise zwei Metaboliten durch ein Wassermolekül $\left(\mathrm{H}_{2} \mathrm{O}\right)$ ist die Differenz 18.0106 Dalton. Die neu entdeckte Metabolitengruppe (Kreise in Magenta und Cyan mit gelben Verbindungen) haben das gleiche Grundgerüst, aber unterscheiden sich durch verschieden lange Fettsäuren (Kreise 1, 4, 5 in Magenta) bzw. fehlende (Kreis 7 in Cyan) Fettsäure. Deren MALDI-MSI- und FISH-Bilder zeigen, dass die Metaboliten mit Fettsäuren mit den symbiontischen Bakterien überlagerten, während der Metabolit ohne Fettsäure mit dem Gewebe des Wirts übereinstimmt. 
diesen Zellen mit FISH identifizieren. Das Potenzial dieses korrelativen Verfahrens namens metaFISH (metabolite- and FISH imaging) zeigten wir anhand von Gewebe einer nicht-kultivierbaren Bakterien-Tier-Symbiose aus der Tiefsee.

Eine automatisierte korrelative Analyse auf Basis der Kolokalisierung der Bilddaten ermöglicht, Metabolitensignaturen symbiotischem und nicht-symbiotischem $\mathrm{Ge}$ webe statistisch zuzuordnen. Damit bietet metaFISH die Grundlage, Metaboliten ihren Produzenten in symbiotischen Organismen zuzuordnen.

Die verbesserte räumliche Auflösung zeigt, dass das Metabolom der intrazellulären Symbiontengemeinschaften zwischen Wirtszellen variiert, möglicherweise als Reaktion auf lokale Mikro-Umgebungen (Abb. 2).

Die Integration der metaFISH-Daten in chemische Netzwerke schafft einen Zusammenhang zwischen Chemie und Verteilung der Metabolite (Abb. 3). In den Netzwerken fanden wir eine Gruppe mit acht chemisch verwandten Metaboliten, von denen sechs unserer initialen Bildanalyse entgingen. Jeder Punkt des Netzwerks repräsentiert ein Metabolitenbild. Das metaFISH-Netzwerk zeigt, dass je nach chemischer Modifikation die acht Metaboliten räumlich mit symbiotischen und nicht-symbiotischen Geweben korrelieren, was auf eine chemische Interaktion der Partner hindeuten könnte.

\section{Auflösung ist nicht alles}

Angespornt durch die neuen Erkenntnisse, arbeiten wir nun auf subzelluläre Auflösungen mittels MALDI-FISH hin. Ein experimentelles Setup erreichte bereits eine Auflösung von 0,6 Mikrometern pro Pixel [11] und stellt einen wichtigen Schritt für MALDI-MSI einzelner Bakterien dar. Dennoch bleiben korrelative Mikroskopietechniken wie FISH, selbst bei Methoden mit subzellulärer Auflösung [12], essenziell für die Analyse chemischer Bilddaten. Um die chemische Sprache zwischen mehrzelligen Organismen und
Bakterien zu entschlüsseln, bieten korrelative Ansätze wie MSI und FISH einzigartige Möglichkeiten zur Visualisierung dieser Interaktionen.

\section{Danksagung}

Wir danken unseren Kollaborationspartnern und der Max-Planck-Gesellschaft, dem MaxPlanck-Institut für Marine Mikrobiologie und allen Mitgliedern der Abteilung Symbiose und der Gruppe Metabolic Interactions. Wir danken auch Fanni Aspetsberger und Katrin Matthes für die Kommentare zum Text. Außerdem bedanken wir uns bei Nicole Dubilier für die großzügige Förderung der Arbeiten durch Projekte der Betty and Gordon Moore Foundation und dem ERC.

\section{Literatur}

[1] Cleary JL, Condren AR, Zink KE et al. (2017) Calling all hosts: bacterial communication in situ. Chem 2:334-358 [2] Caprioli RM (2015) Imaging mass spectrometry: enabling a new age of discovery in biology and medicine through molecular microscopy. J Am Soc Mass Spectrom 26:850-852 [3] Zhou Q, Guevara CR, Hopf C (2018) Massenspektrometrie in der Biomedizin- und Pharmaforschung. BIOspektrum 24:694-696

4] Alexandrov T, Ovchinnikova K, Palmer A et al. (2019) METASPACE: A community-populated knowledge base of spatial metabolomes in health and disease. bioRxiv:539478 [5] Perry WJ, Spraggins JM, Sheldon JR et al. (2019) Staphylococcus aureus exhibits heterogeneous siderophore production within the vertebrate host. Proc Natl Acad Sci USA 116:21980-21982
[6] Amann R, Fuchs BM (2008) Single-cell identification in microbial communities by improved fluorescence in situ hybridization techniques. Nat Rev Microbiol 6:339-348 [7] Kaltenpoth M, Strupat K, Svatos A (2016) Linking metabolite production to taxonomic identity in environmental samples by (MA)LDI-FISH. ISME J 10:527-531

[8] Rappez L, Stadler M, Triana S et al. (2019) Spatial singlecell profiling of intracellular metabolomes in situ. bioRxiv:510222

[9] Geier B, Sogin EM, Michellod D et al. (2020) Spatial metabolomics of in situ host-microbe interactions at the micrometre scale. Nat Microbiol 5:498-510

[10] Kompauer M, Heiles S, Spengler B (2017) Atmospheric pressure MALDI mass spectrometry imaging of tissues and cells at 1.4- $\mu \mathrm{m}$ lateral resolution. Nat Methods 14:90-96

[11] Niehaus M, Soltwisch J, Belov ME et al. (2019) Transmission-mode MALDI-2 mass spectrometry imaging of cells and tissues at subcellular resolution. Nat Methods 16:925-931

[12] Decelle J, Veronesi G, Gallet B et al. (2020) Subcellular chemical imaging: new avenues in cell biology. Trends Cell Biol 30:173-188

Funding Open Access funding provided by Projekt DEAL.

Open Access Dieser Artikel wird unter der Creative Commons Namensnennun 4.0 International Lizenz veröffentlicht, welche die Nutzung, Vervielfältigung, Bearbeitung, Verbreitung und Wiedergabe in jeglichem Medium und Form ordnungssem ̈̈ B en/die urspring zur Cretive Cond die Quelle angeben, ob Änderungen vorgenommen wurden. Die in diesem Artikel enthaltenen Bilder und sonstiges Drittmaterial unterliegen ebenfalls der genannten Creative Commons Lizenz, sofern sich aus der Abbildungslegende nichts anderes ergibt. Sofern das betreffende Material nicht unter der genannten Creative Commons Lizenz steht und die betreffende Handlung nicht nach gesetzlichen Vorschriften erlaubt ist, ist fur die oben aufgefuhrten Weiterverwendungen des Materials die Einwilligung des jeweiligen Rechteinhabers einzuholen. Weitere Details zur Lizenz entnehmen Sie bitte de

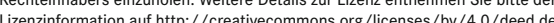

\section{Korrespondenzadresse:}

Dr. Manuel Liebeke

Max-Planck-Institut für Marine Mikrobiologie

Celsiusstraße 1

D-28359 Bremen

mliebeke@mpi-bremen.de

Benedikt Geier
$\begin{aligned} & 2010-2015 \text { Biologiestudium (B. Sc. und M. Sc.) an der LMU München. 2015-2020 } \\ & \text { Promotion am Max-Planck-Institut für Marine Mikrobiologie, Abteilung Symbiose, } \\ & \text { Bremen, bei Prof. Dr. N. Dubilier unter direkter Betreuung von Dr. M. Liebeke. Seit } \\ & \text { Mai 2020 Postdoktorand in der Gruppe Metabolic Interactions am MPI Bremen. }\end{aligned}$
$\begin{aligned} & \text { Manuel Liebeke } \\ & \text { 2001-2006 Studium der Pharmazie und 2010 Promotion an der Universität Greifs- } \\ & \text { wald. 2010-2013 Postdoc-Aufenthalt am Imperial College, London, UK, in der Abtei- } \\ & \text { lung Systems Medicine. Seit 2013 am Max-Planck-Institut für Marine Mikrobiologie, } \\ & \text { seit 2018 Leiter der Gruppe Metabolic Interactions in der Abteilung Symbiose. }\end{aligned}$

